\title{
Riesgo de adquirir enfermedades crónicas no transmisibles provocadas por sedentarismo, de los empleados de la Universidad Pedagógica Nacional Francisco Morazán
}

\author{
Ramón Antonio Vega \\ Universidad Pedagógica Nacional Francisco Morazán
}

\section{Introducción}

Está científicamente comprobado que la buena condición física y fisiológica tiene una relación directa con la composición corporal, y esta a la vez, con los hábitos de vida y el creciente sedentarismo de la sociedad moderna. La relación anterior, ha venido provocando un debilitamiento progresivo del funcionamiento fisiológico del organismo humano, según dijo Getchel 1990; 22, exponiéndolo al riesgo de adquirir, enfermedades que impiden el desempeño eficiente de las funciones corporales, y en el caso de los empleados de la universidad, de sus respectivas funciones laborales.

La automatización y el desarrollo acelerado de nuestra sociedad, así como, la práctica de hábitos nocivos para la salud y la inactividad física han incrementado la prevalencia de enfermedades cardiovasculares, la hipertensión, los accidentes cerebro vascular, la diabetes mellitus tipo II y algunos tipos de cáncer. Según la Organización Mundial de la Salud, en 1999, la prevalencia de estas enfermedades era de un $60 \%$ del total de muertes en el mundo y se estima que para el año 2020 ascenderá a un $73 \%$. y según la O.P.S.,2002, pag.123, la prevalencia actual de enfermedades crónicas no transmisibles en los países en vías de desarrollo, es de un 77\%, afectando mayormente a los adultos en su época más productiva.

Uno de los factores más importantes en el desarrollo de estas 
enfermedades es la inactividad física, la cual representa aproximadamente un $77.5 \%$ en los Estados Unidos, planteamiento que hace la OPS 2002, pag.123. Y esta cifra llega a aumentar hasta un $80 \%$ o un $90 \%$ en algunos países suramericanos como Chile, Perú y Argentina según estudios realizados por Casen y Saben 2000, pag. 33. Honduras no está lejos de esos números si consideramos que actualmente hay en nuestro país 66,000 personas con Diabetes Mellitus, información publicada por el Dr. Marco Sarmiento en el diario La Tribuna 23 Nov., 2002, pag.42 y hay una alta prevalencia de niños obesos, según dicho diario, en su publicación del día 12 de Enero, 2003, pag. 48.

En los últimos años los empleados de la Universidad Pedagógica Nacional Francisco Morazán se han visto afectados por la falta de actividad física debido al tipo de trabajo sedentario que la mayoría realiza, lo que se expresa en frecuentes incapacidades y a veces hasta muerte, de algunos de sus compañeros, provocados por enfermedades crónicas no transmisibles, en algunos de estos casos dicha enfermedad se pudo haber evitado, con un poco de orientación sobre los beneficios que la actividad física y la correcta alimentación tienen sobre la salud.

Es importante para los empleados mencionados, mantener el buen funcionamiento fisiológico del cuerpo y reducir el riesgo de adquirir enfermedades crónicas no transmisibles, como la diabetes mellitus tipo II, enfermedades coronarias, obesidad, enfermedades cardiovasculares, enfermedades cerebrovasculares, etc. Garantizando con esto una mejoría sustancial en sus niveles de salud y una oportunidad de aumentar sus expectativas de vida, en una sociedad donde cada día es más frecuente la presencia de patologías relacionadas con el estilo de vida, tal es la apreciación de Alexander, 1999 pag 40.

La implementación de un programa sistemático de actividad física para los trabajadores como política institucional vendría a reducir el riesgo de mortalidad provocado por enfermedades crónicas no transmisibles, mejorar la calidad de vida, aumentar la longevidad, así como, la eficiencia física y psicológica de los empleados de la UPNFM. 
Riesgo de adquirir enfermedades crónicas no transmisibles provocadas por sedentarismo, de los empleados de la Universidad Pedagógica Nacional Francisco Morazán

\section{Objetivos}

1. Determinar el nivel de obesidad y sobrepeso de los empleados de la Universidad Pedagógica Nacional Francisco Morazán.

2. Determinar el nivel de actividad física que realizan los empleados de la UPNFM, en sus actividades cotidianas.

3. Establecer el riesgo de adquirir enfermedades cardiovasculares, obesidad o diabetes mellitus tipo II, de los empleados de la UPNFM, considerando su porcentaje de grasa y nivel de actividad física.

4. Mostrar la relación entre el sedentarismo y el riesgo de padecer enfermedades crónicas no transmisibles, entre los empleados de la UPNFM

\section{Diseño Metodológico}

El presente estudio relacional descriptivo pretendo, como su nombre lo indica, establecer y describir la relación que factores como el sedentarismo, y la composición corporal tienen con el riesgo de adquirir enfermedades cardiovasculares, obesidad o diabetes Mellitus tipo II, en los empleados de la UPNFM.

Para lograr lo anterior se utilizaron, cuestionarios orientados a recabar información sobre la actividad física realizada por los empleados de la UPNFM, instrumento que determine su nivel de sedentarismo, y mediciones antropométricas (peso, talla, porcentaje de grasa), además se medirán la circunferencia de la cintura, a la altura de la cicatriz umbilical, y la circunferencia de la cadera a la altura del trocante, utilizando esa información para determinar la forma corporal de los sujetos, para clasificarlo según Larson 1984, o Bray 1992 y determinar el riesgo de adquirir Diabetes Mellitus tipo II, obesidad y enfermedades cardiovasculares.

\section{Población y muestra}

La población del estudio son los empleados de la UPNFM que laboran en el sistema presencial de Tegucigalpa, mientras que la muestra fue el $22.31 \%$ de los empleados con acuerdo de empleo de tipo permanente, las mediciones se realizaron considerando diferentes estratos: docentes, administrativos y de servicio, etc. Por lo anterior 
el tipo de muestra a utilizar fue probabilística estratificada.

\section{Técnicas de recolección de datos}

Para recolectar la información general pertinente a la investigación se utilizó un cuestionario internacional de actividad física IPAQ: Formato corto autoadministrado de los últimos 7 días, con preguntas cerradas y la escala de medición de factores de riesgo elaborada por la Michigan Heart Association, reconocida por la organización mundial de la salud, modificadas y adaptadas a nuestro contexto y en un solo instrumento, para obtener información general de los sujetos a estudiar, así como sus niveles de sedentarismo, y luego se hicieron las mediciones antropométricas necesarias, basándonos en el protocolo de faulkner para el porcentaje de grasa y el índice de cintura-cadera, que ha sido utilizado de manera convencional para determinar distribuciones de grasa corporal tipo Androgénica (tipo II) y Ginecoide (tipo IV) utilizados por Bray, (1992), y Larson, (1984, pag. 36 )

El protocolo de faulkner para el porcentaje de grasa tiene la siguiente fórmula para su desarrollo: Sumatoria de 4 pliegues cutáneos x 0.153 $-5.783$

\section{Plan de Análisis}

- Una vez obtenida la información, producto de las mediciones antropométricas realizadas, se procedió a la aplicación de las fórmulas matemática pertinentes, para el análisis de los datos. Para determinar el porcentaje de grasa se midieron 4 pliegues cutáneos. Tríceps, Subescapular, Suprailíaco y Abdominal, luego se les dio tratamiento matemático utilizando la formula de Faulkner (1983), para determinar al porcentaje de grasa en el cuerpo de los sujetos de estudio

- Obtenida la información del instrumento teórico aplicado, se procedió a darle el tratamiento estadístico pertinente para el análisis de la información recopilada.

- Analizados los dos tipos de información se procedió, basados en parámetros internacionales, a correlacionar las variables del estudio para determinar el riesgo de adquirir enfermedades crónicas no trasmisibles de los sujetos de estudio, tanto por su composición corporal, sus antecedentes personales, como por 
Riesgo de adquirir enfermedades crónicas no transmisibles provocadas por sedentarismo, de los empleados de la Universidad Pedagógica Nacional Francisco Morazán

su nivel de sedentarismo.

- Una vez analizadas y relacionadas las variables se estructuraron las conclusiones del estudio.

\section{Definición de variables}

Para el presente estudio se consideraron las variables indicadas en la tabla \# 1 presentada a continuación:

\section{Tabla de definición de variables}

\begin{tabular}{|l|l|}
\hline \multicolumn{1}{|c|}{ Variables } & \multicolumn{1}{c|}{ Definición } \\
\hline Sedentarismo & $\begin{array}{l}\text { Condición humana donde el individuo } \\
\text { no realiza actividad física de manera } \\
\text { sistemática, con la frecuencia, ni la } \\
\text { intensidad necesaria para estimular un } \\
\text { cambio fisiológico en el organismo. }\end{array}$ \\
\hline $\begin{array}{l}\text { Riesgo de padecer } \\
\text { enfermedades crónicas } \\
\text { no transmisibles }\end{array}$ & $\begin{array}{l}\text { Es la posibilidad que tiene cada individuo } \\
\text { de adquirir o desarrollar, alguna de las } \\
\text { siguientes enfermedades: obesidad, } \\
\text { enfermedades cardiovasculares, diabetes } \\
\text { mellitus tipo II. }\end{array}$ \\
\hline Obesidad & $\begin{array}{l}\text { Se refiere a la cuantificación de tejido } \\
\text { adiposo subcutáneo localizado en } \\
\text { diferentes puntos anatómicos. }\end{array}$ \\
\hline Factor genético & $\begin{array}{l}\text { Antecedentes de padecimiento de } \\
\text { enfermedades crónicas en familiares } \\
\text { directos de los sujetos de la muestra. }\end{array}$ \\
\hline
\end{tabular}

\section{Resultados}

Luego de aplicar el instrumento, podemos notar que el $55.42 \%$ de los encuestados no realiza ninguna actividad física vigorosa, y apenas un $25.3 \%$ realiza alguna actividad vigorosa más de 3 días por semana, si sumamos los datos que van de 3 a 7 días, frecuencia que es lo teóricamente recomendable, para el logro de algún nivel de estimulación fisiológico, que provoque alguna mejora en el organismo. Esto lo podemos observar en el gráfico \#1 que se presenta a continuación y que ilustra sobre el nivel de sedentarismo que existe entre los empleados de la Universidad Pedagógica Nacional Francisco Morazán. 
Considerando la realización de actividad física de manera moderada o con un nivel de exigencia inferior al del grupo anterior, podemos notar en el gráfico \# 2, que un $56.63 \%$ contestò que no realiza dicha actividad ningun dia a la semana, y si se suman los sujetos que realizan actividad física moderada entre 1 y 4 dias a la semana apenas, se alcanza un $28.91 \%$ mientras que el resto de los encuestados realizan actividad física moderada entre 5 y 7 dias a la semana representando solamente el $12.04 \%$ de la muestra.La información anterior se puede entender de manera más tangible al observar, las diferencias entre las barras, de los sujetos de la muestra que realizan actividad física de manera moderada, y los que no realizan actividad física de ninguna sistematica.

En contraste con la actividad física moderada o vigorosa, realizada por los sujetos de la muestra, que se puede cuantificar en minutos, el tiempo en que permanecen

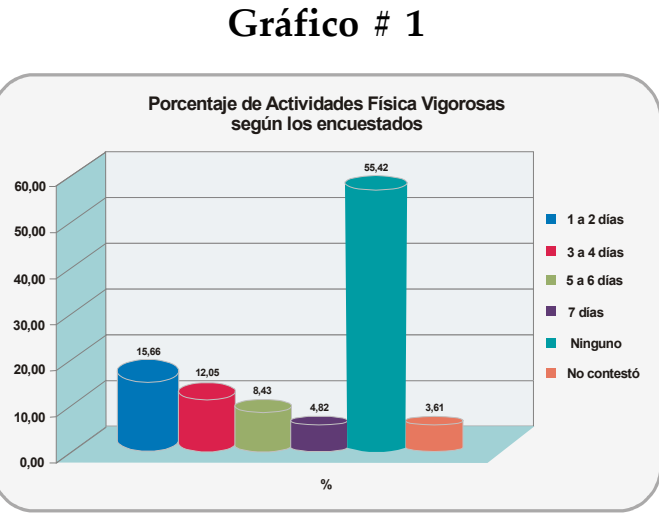

Gráfico \# 2

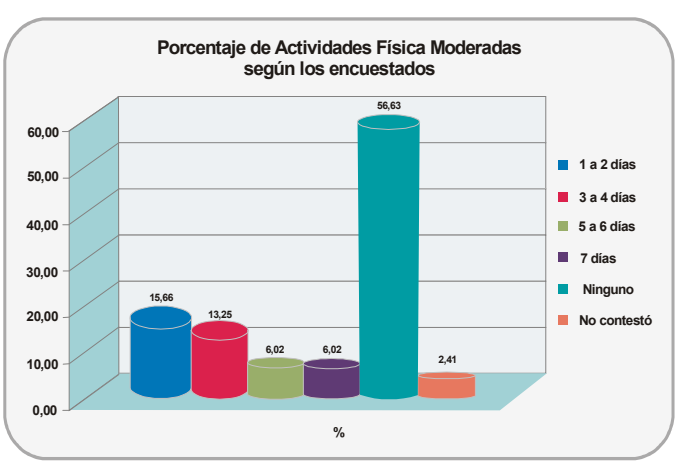

Gráfico \# 3

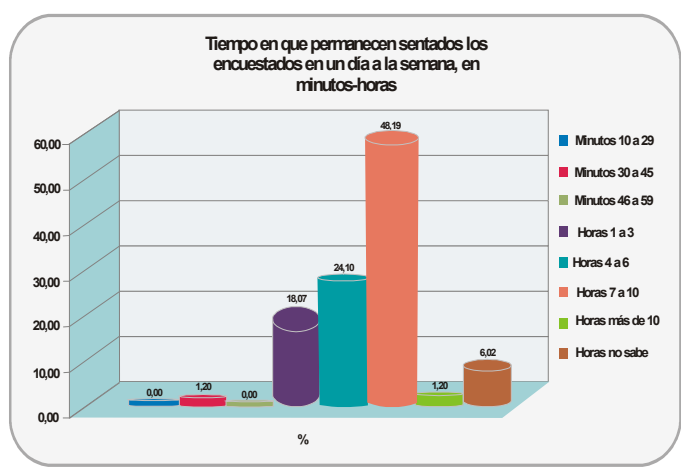
sentados, en un dia regular, se mide en horas, tal y como se aprecia a continuación en el gráfico \# 3, tanto así que considerando las barras del grafico que representan los sujetos que permanecen sentados más 
Riesgo de adquirir enfermedades crónicas no transmisibles provocadas por sedentarismo, de los empleados de la Universidad Pedagógica Nacional Francisco Morazán

de 4 horas en el dia, ese encuentra que el $72.19 \%$ de los sujetos de la muestra permanecen en esa posición entre 4 y 10 horas, tomando en cuenta el tiempo de trabajo y el tiempo de permanencia en su hogar, datos que indican el alto nivel de sedentarismo de los empleados de la UPNFM, en perjuicio de su salud, adicionalmente, según las conclusiones del estudio sobre factores de riesgo realizado por la Organización Mundial de la Salud (OMS), en el año 1999, el modo de vida sedentaria es una de las 10 causas fundamentales de mortalidad y discapacidad en el mundo (AMDCO, 2002:2).

La situación sobre la Gráfico \# 4

obesidad, planteada anteriormente, alcanza niveles alarmantes al observar la información contenida a continuación en el grafico \# 4 donde se observa con claridad, el alto porcentaje $(51.81 \%)$ de empleados con un sobrepeso entre 2.3 kilos

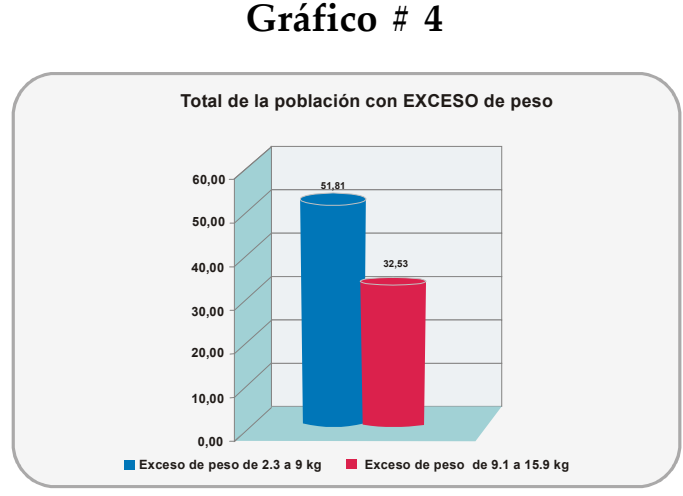
(5.06 libras) y 9 kilos (19.8 libras) y notamos también como un 32.53 $\%$ tienen un grave sobrepeso que va desde los 9.1 kilos (19.8 libras) hasta los 15.9 kilos (34.98 libras), alcanzando con esto niveles de obesidad en distintos grados, que los puede llevar a adquirir otras enfermedades como diabetes mellitus tipo II y enfermedades cardiovasculares relacionadas. Si se suma la información de ambas barras del gráfico es fácil darse cuenta que un $84.34 \%$ de la población de los empleados de la UPNFM, se encuentra en riesgo real de adquirir enfermedades crónicas no transmisibles, provocadas en primera instancia por sedentarismo.

La mayoría de las enfermedades crónicas presentan una base genética, si uno de los progenitores ha sufrido una dolencia cardíaca, un cáncer o diabetes mellitus, el hijo tendrá una mayor probabilidad de contraer una de esas enfermedades, pero los factores del entorno contribuyen a su desarrollo y posible progresión, tal es el planteamiento hecho por Williams en el 2002. Considerando que los antecedentes familiares juegan un papel importante en la adquisición de enfermedades crónicas no transmisibles, se procedió a indagar en ese sentido, 
encontrando que solamente el $34.94 \%$ de los empleados de la UPNFM no tienen ningún historial familiar relacionado con dolencias cardiovasculares, lo que lleva a ubicarse ante un $65.06 \%$ con posibilidad genética de padecer de ese tipo de enfermedades, situación que se agrava si se

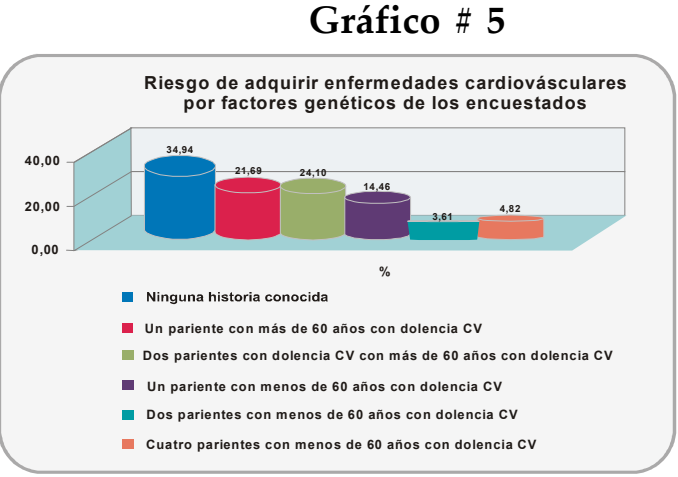

suman los casos de sujetos con familiares menores de 60 años, lo que da un $22.89 \%$ con alta propensión a padecer este tipo de enfermedad, sin importar la edad, cuando regularmente las enfermedades cardiovasculares son características en el adulto mayor. Información que se ilustra en el grafico \# 5 .

\section{Conclusiones}

Luego de haber analizado los resultados obtenidos en la presente investigación y haciendo relación con los objetivos del estudio se puede concluir lo siguiente:

En relación al porcentaje de grasa de los empleados de la muestra, se encontró que hay una alta cantidad de los mismos (84.34\%) que tienen porcentajes de grasa por encima de los parámetros normales recomendados para personas sedentarias, lo que implica que los niveles de sobrepeso entre los empleados de la UPNFM, son un claro indicador y un campo abierto para una probable epidemia de obesidad en la institución.

Dichos niveles de sobrepeso están en directa relación con los niveles de actividad física, realizada en promedio por parte de los empleados, pues es evidente en el estudio, que las actividades cotidianas realizadas por los mismos, tanto en su horario laboral, como en su tiempo libre, son de exigencia física insuficiente como para estimular una mejoría en los procesos fisiológicos del organismo, convirtiéndolos en sujetos sedentarios y expuestos a una serie de riesgos producto de esa condición. 
Riesgo de adquirir enfermedades crónicas no transmisibles provocadas por sedentarismo, de los empleados de la Universidad Pedagógica Nacional Francisco Morazán

Condición que podría considerarse preocupante en la UPNFM, pues la cantidad de personas que no hacen ninguna actividad física moderada y mucho menos vigorosa, predomina en un alto porcentaje sobre aquellos que de alguna manera se ven medianamente exigidos físicamente en sus actividades cotidianas o tienen como hábito vital, el hacer ejercicio de manera sistemática como medida preventiva y de mantenimiento de la salud.

El estilo de vida sedentario, caracterizado por el tiempo promedio en que los empleados permanecen sentados en un día regular y el sobrepeso, marcado por los porcentajes de grasa, los convierte en blanco perfecto para todas aquellas enfermedades crónicas no transmisibles, especialmente aquellas enfermedades metabólicas relacionadas con la obesidad, como diabetes mellitus tipo II o enfermedades cardiovasculares.

Como se pudo observar en la fundamentación teórica de este estudio, la obesidad es el principal factor desencadenante del riesgo de adquirir enfermedades como las antes mencionadas, pues en directa relación con el sedentarismo crean en el individuo las condiciones propicias para el desarrollo de las mismas, situación que suele agravarse por los antecedentes familiares relacionados con éstas enfermedades.

En la UPNFM, según el estudio, se juntan entre la población de empleados docentes y administrativos, los tres factores antes mencionados: sedentarismo, obesidad y herencia, lo que plantea una situación de epidemia latente de enfermedades crónicas no transmisibles, como diabetes tipo mellitus tipo II y enfermedades cardiovasculares, en un alto nivel, lo que podría, en un futuro cercano, agravar la situación laboral de los empleados de la institución, obligándolos a reducir el rendimiento laboral, causado por el padecimiento de este tipo de enfermedades, provocando la solicitud de constantes permisos, incapacidades, largos procesos de recuperación y hasta la muerte prematura, o en el mejor de los casos deterioro severo de su calidad de vida.

Las afirmaciones anteriores se entienden fácilmente si se logra establecer la relación de los factores vinculantes, de la siguiente forma: El sedentarismo caracterizado por la falta de actividad física produce obesidad, la obesidad caracterizada por el exceso de grasa en el cuerpo, 
crea las condiciones metabólicas apropiadas para el desarrollo de la diabetes mellitus tipo II en los individuos que poseen propensión genética, la diabetes mellitus tipo II es una enfermedad altamente relacionada con las enfermedades cardiovasculares y relacionándola con la obesidad crean una condición de alto riesgo para que se produzcan en el individuo enfermedades cardiovasculares como, cardiopatía isquémica, insuficiencia cardiaca congestiva e hipertensión arterial; sumado al cuadro de historias familiares en esta línea entre los empleados de la UPNFM.

Ante el cuadro descrito anteriormente, se puede afirmar que de acuerdo a los parámetros de riesgo de adquirir enfermedades crónicas no transmisibles, relacionadas con sedentarismo reconocidos por la OMS, los empleados de la UPNFM, se encuentran en un nivel de riesgo alto, tanto por su estilo sedentario de vida, sus niveles de obesidad, como por el factor genético predominante entre los mismos. 
Riesgo de adquirir enfermedades crónicas no transmisibles provocadas por sedentarismo, de los empleados de la Universidad Pedagógica Nacional Francisco Morazán

\section{Bibliografía}

Alexander, P.(1999) Características Físicas y Morfológicas del Venezolano, en Memoria del IV Congreso Nacional de Educación Física, Deporte y Recreación. Fuser Caracas, Venezuela.

Getchel, B. (1990) Condición Física. Cómo mantenerse en forma. Editorial Limusa, México.

Bray, G: (1992) Obesity increases risk for diabetes. International Journal of obesity,16 (suppl.4) S13-S17.

OMS, (1999) Prevención y lucha contra las enfermedades Cardiovasculares en la comunidad, Informes técnicos n⿳ำ 732 . Ginebra, Suiza.

OPS (2002) La inactividad física, un factor principal de riesgo para la salud en las Américas. Washington, Estados Unidos. 